\title{
Mezclas vítreas de material reciclado con decoración digital MOSAINK
}

\author{
V. FAUBEL-SERRA ${ }^{1}$, J. ROMERO-VALIENTE ${ }^{2}$ \\ ${ }^{1}$ Ldo. Químicas I. Cerámica. (TOGAMA).Onda.Castellón.España. \\ ${ }^{2}$ Técnico Mantenimiento Electromecánico. (TOGAMA). Onda.Castellón. España.
}

Este proyecto ha sido presentado a los premios Alfa de Oro en la Feria Internacional CEVISAMA VALENCIA 2011

\begin{abstract}
El mosaico vítreo es un producto clásicamente empleado en piscinas, y limitado a zonas de agua. En esta ocasión se ha querido darle un enfoque completamente diferente, aprovechando las características técnicas del producto; ecológico (materia prima reciclada), no contaminante (emisión nula de gases), alta resistencia a la abrasión, porosidad nula, resistente a productos químicos, resistente a heladas, alto brillo y modularidad.

Como base del proyecto se ha empleado, un producto ya desarrollado con anterioridad en nuestros centros de investigación, al cual se le ha dado un nuevo desarrollo para la obtención de un mosaico vítreo, con unas características técnicas específicas. Habiendo desarrollado un producto vítreo por prensado de alta translucidez, al cual cabía la posibilidad de realizar múltiples coloraciones en seco, se retoma el proyecto con nuevas mezclas de vidrio para poder aplicar la tecnología de la decoración digital.

La unión de estos dos procesos permite desarrollar un producto idóneo para la decoración, iluminación, domótica, sistemas de control en condiciones extremas, como infinidad de nuevos campos de aplicación.
\end{abstract}

Palabras clave mosaico translucido, prensado, decoración digital.

Recycled glass mixes for digital decoration. Mosaik

Being glass mosaic until now a product classically used in swimming pools, as well as in a few other aquatic environments, it was our aim to give it a completely different approach, by using the technical advantages of our product; ecological (recycled raw material), nonpolluting (null gas emission), high resistance to abrasion, zero porosity, resistance to chemical products and stains, frost proof, high brightness and modularity.

As basis of this project, we have used a product we had already developed in our research centers. This product had to be re-formulated, in order to obtain a glass mosaic with specific technical characteristics.

Although we had a vitreous product by high translucency pressing, which we could dye in dry status, we retook the project in order to develop new glass mixtures able to admit the application of the digital decoration technology.

Unifiying these two procedures allowed us to obtain a product suitable for decoration, illumination, home automation, control systems under extreme conditions and many new application fields.

Key words: high translucency, pressed mosaic, digital decoration.

\section{OBJETIVOS.}

El objetivo principal de este proyecto es el diseño y desarrollo de una nueva gama de productos pensados para integrar la tecnología de decoración digital en los mosaicos translucidos por prensado, dotando así de un mayor valor añadido al nuevo producto y creando un concepto nuevo. Se deberá estudiar las características técnicas y composición necesarias del mosaico para que sin perder la translucidez, pueda ser susceptible de poder realizar una decoración digitalizada.

El desarrollo de un nuevo tipo de mosaico vítreo que permita la integración de la decoración digital implicará el rediseño del proceso productivo.

\subsection{Objetivos técnicos específicos del proyecto.}

El vidrio es un material duro, frágil y transparente que ordinariamente se obtiene por fusión a temperaturas cercanas a $1.500{ }^{\circ} \mathrm{C}$ de compuestos vitrificantes, arena de sílice $\left(\mathrm{SiO}_{2}\right)$, fundentes, carbonato sódico $\left(\mathrm{Na}_{2} \mathrm{CO}_{3}\right)$, y estabilizantes, caliza $\left(\mathrm{CaCO}_{3}\right)$.

La llama debe alcanzar una temperatura muy elevada y por eso el aire de combustión se calienta antes de que llegue a los quemadores. La mezcla se funde (zona de fusión) a temperaturas entre $1450-1500{ }^{\circ} \mathrm{C}$ y avanza hacia la zona de enfriamiento, donde tiene lugar el recocido.

En este caso el vidrio utilizado como materia prima, procede del reciclado, mediante su combinación con diferentes productos, se puede desarrollar un mosaico capaz de ser decorado digitalmente. 


\subsection{Decoración del proyecto.}

En este proyecto se utilizó una maquina de decoraciór digital por inyección de tintas cerámicas que permiter decorar cualquier pieza a una velocidad máxima, combinandc diferentes colores, imprime una velocidad de 50 metros po 60.00 minuto, sin necesidad de detener la pieza y decora cualquie superficie sin tener contacto con la pieza, lo que evita todos los defectos provocados por este rozamiento.

Para gestionar el proceso de impresión de inyección $\mathrm{d} \epsilon$ tinta cada cabezal tiene su propio controlador dedicado. Otros 20,00 circuitos incluidos son convertidores A/D y D/A, SDRAN. y memoria flash, y un chip Ethernet PHY que permite, c la tarjeta, comunicarse con la procesador principal de lá impresora.

El desarrollo de los nuevos mosaicos se basa en los actuales productos de TogAma con características técnicas de alta resistencia a la abrasión, productos químicos, heladas y porosidad nula.

Para desarrollar la nueva gama de mosaicos se definen las características del nuevo material vitrificado: temperatura de fusión, facilidad de decoración, absorción de agua, alta integración de los colores, resistencia a la abrasión, transparencia, persistencia en el tiempo, etc.

En su fabricación se emplea como materia prima base, vidrio plano blanco, seleccionando diferentes muestras del mercado de reciclado (Vidrio A y B).

Al partir de un material heterogéneo, es necesario encontrar materiales adicionales que den estabilidad al vidrio, elevando su transparencia, sean fácilmente compactables y posean un amplio palier de cocción.

Esta compactación ha sido uno de los talones de Aquiles del proyecto, pues por una parte debíamos encontrar un compromiso entre la translucidez del material, la resistencia mecánica de las piezas en crudo para poder ser manipulables y la porosidad en superficie para una integración completa de los colores en las fichas.

Establecemos las granulometrías de los diferentes materiales empleados.
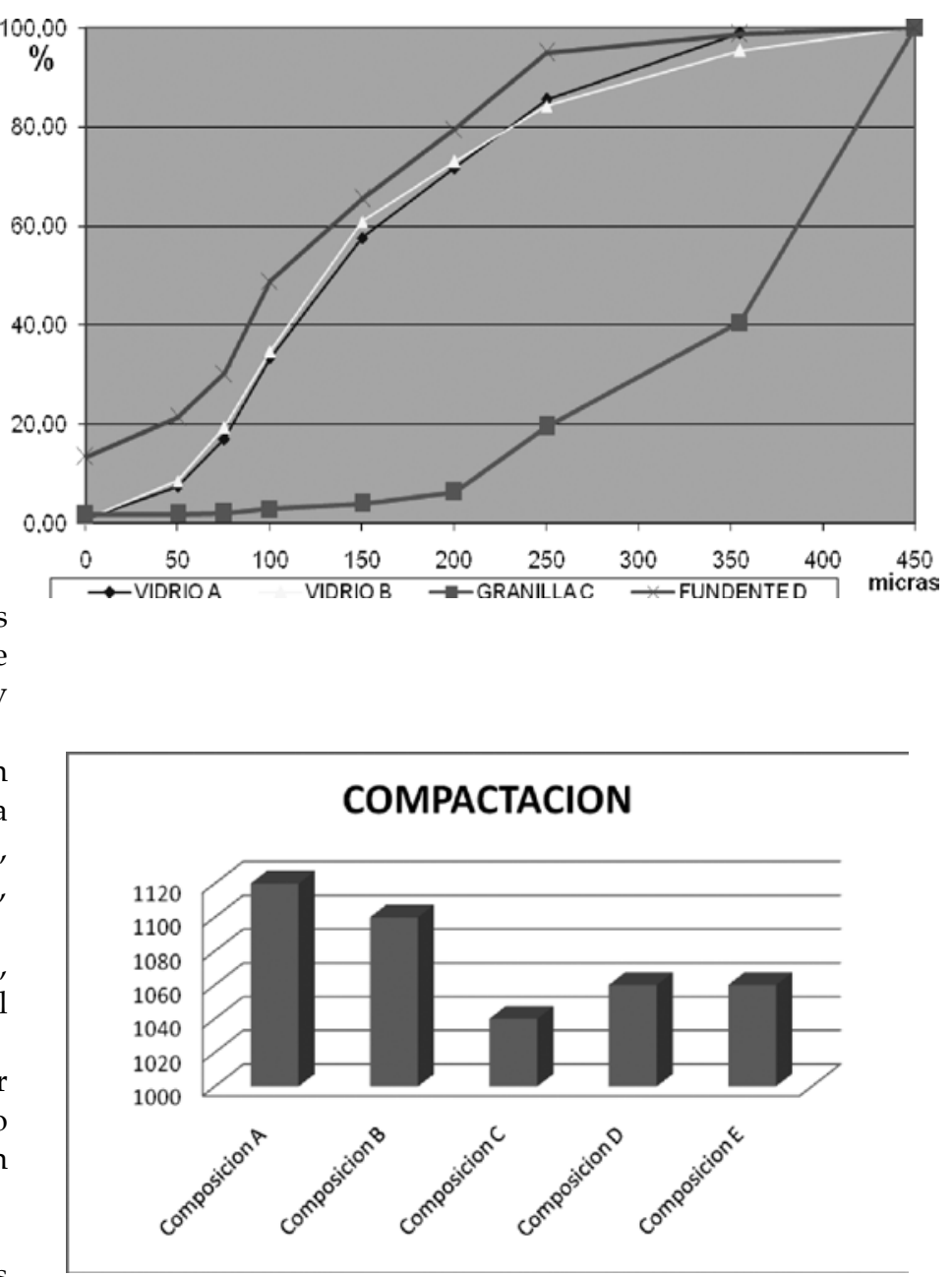

Se han realizado ensayos de compactación de las diferentes mezclas, para obtener una resistencia mecánica en crudo elevada, pues si bien la decoración no se aplica directamente sobre el material prensado o ficha, ésta debe ser manipulable. Eliminamos las composiciones A y B, continuando el desarrollo con las composiciones C, D y E.

\begin{tabular}{|c|c|c|c|c|}
\hline & L & A & B & Ind. Blancura \\
\hline BLANCO STD EQUIPO & 98,55 & 0,14 & 0,56 & 98,73 \\
\hline FICHA D & 86,14 & 2,31 & 3,17 & 53,15 \\
\hline FICHA C & 61,25 & 0,31 & 0,09 & 28,92 \\
\hline FICHA B & 47,8 & 0,18 & 0,25 & 14,7 \\
\hline FONDO NEGRO & 25,8 & 0,06 & 0,53 & 11,02 \\
\hline
\end{tabular}


Sobre estas composiciones se han realizado los ensayos de resistencia mecánica en verde, pues hay que recordar que en el proceso de producción del mosaico vítreo no existe la etapa de secado, lo cual dificulta la manipulación de las fichas.

Si bien los mejores datos obtenidos ni se aproximan a los valores mínimos de un bizcocho de monoporosa en verde (10$\left.14 \mathrm{Kg} / \mathrm{m}^{2}\right)$, al menos permite realizar la manipulación de las fichas con ciertas garantías.

Una vez seleccionadas las pruebas se realizan medidas colorimétricas por transparencia, situando las probetas sobre un fondo negro estandarizado y midiendo el color, el mejor resultado fue la prueba $\mathrm{D}$. (Tabla 1 ).

Definidos los parámetros de compactación, transparencia, y resistencia mecánica, se deben introducir los parámetros finales de porosidad en crudo, para la penetración e integración plena de los colores cerámicos.

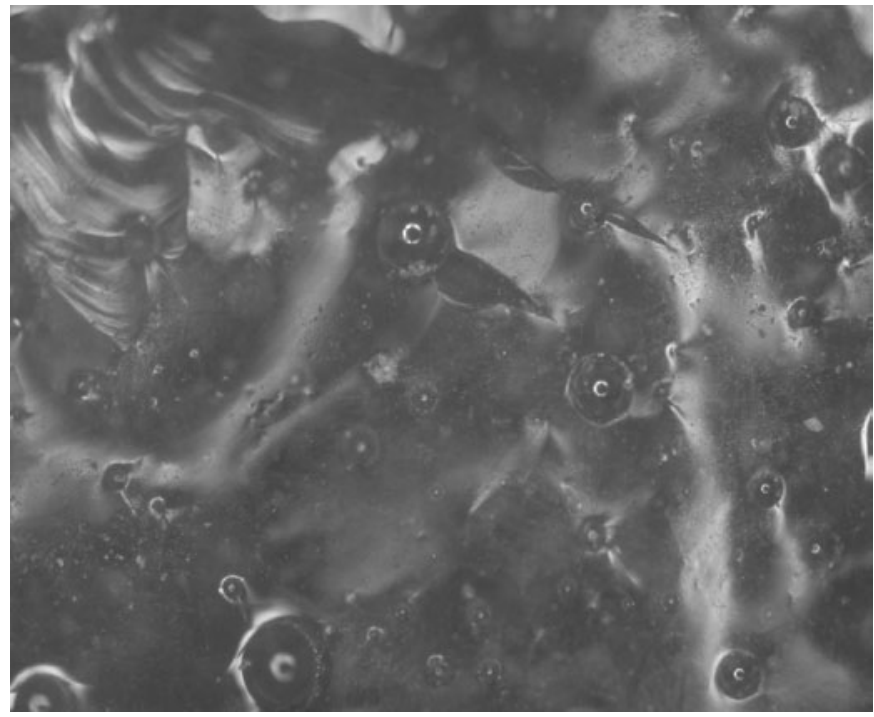

Imagen superficie de una muestra refundida. Microscopía óptica de luz reflejada.

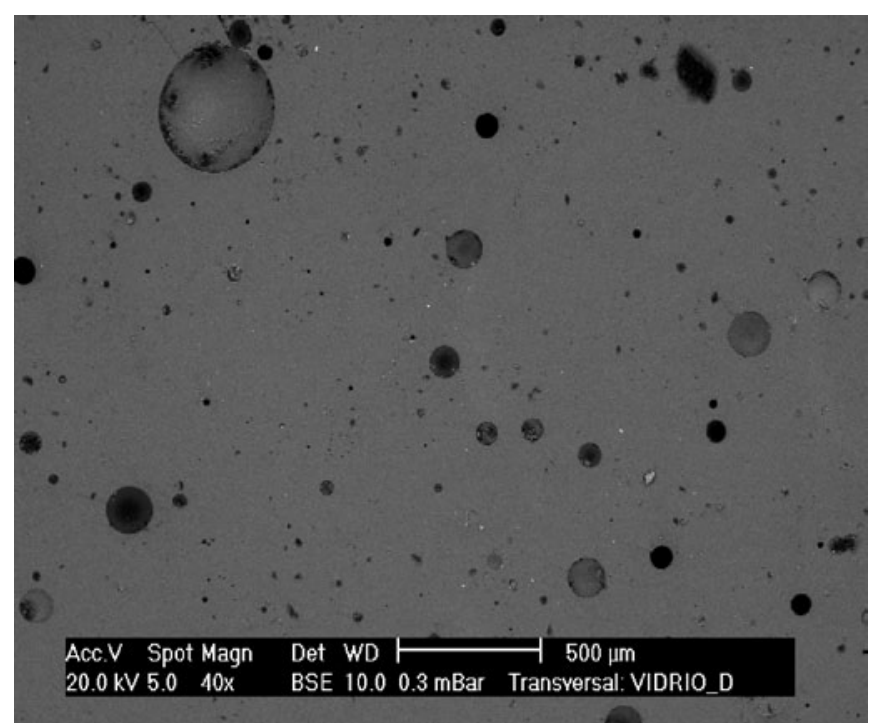

Imagen superficie pulida de una muestra de vidrio sinterizado. Microscopía óptica de luz reflejada.

Bol. Soc. Esp. Ceram. Vidr. Vol 50. 2, marzo-abril 201
Las maquinas existentes en el mercado emplean tintas cerámicas, para una decoración digital de productos a una temperatura de cocción superior a $1080{ }^{\circ} \mathrm{C}$ (monoporosa) hasta $1200{ }^{\circ} \mathrm{C}$ (porcelánico) y ciclos largos de cocción desde $35^{\prime}$ hasta $50^{\prime}$.

Por estas razones las materias primas y productos deben interferir lo mínimo posible en el rendimiento de las tintas cromáticas. De modo que el producto final pueda tener una coloración permanente en el tiempo, resistente a productos químicos y una integración completa dentro del mosaico.

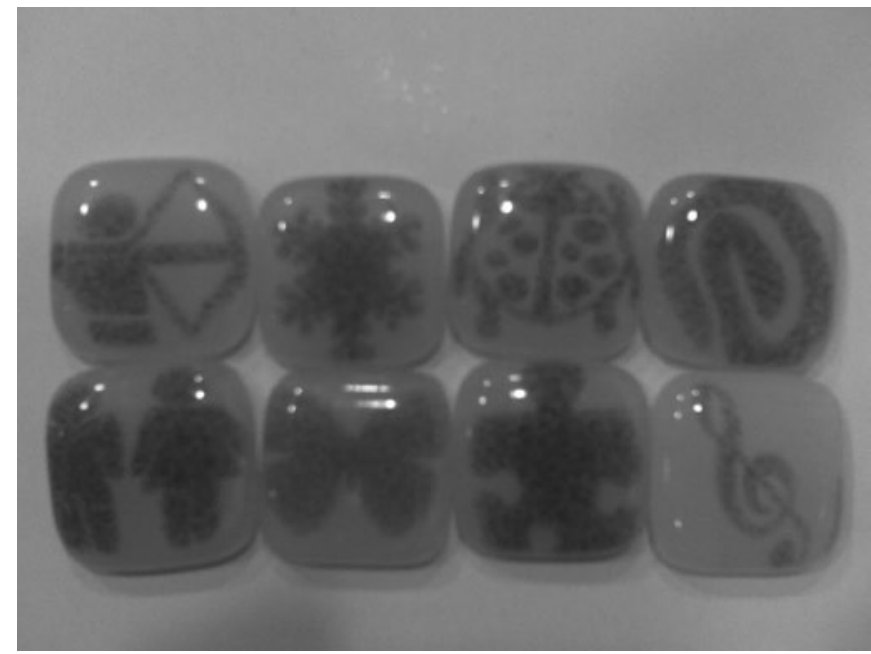

Muestras de las teselas de mosaico

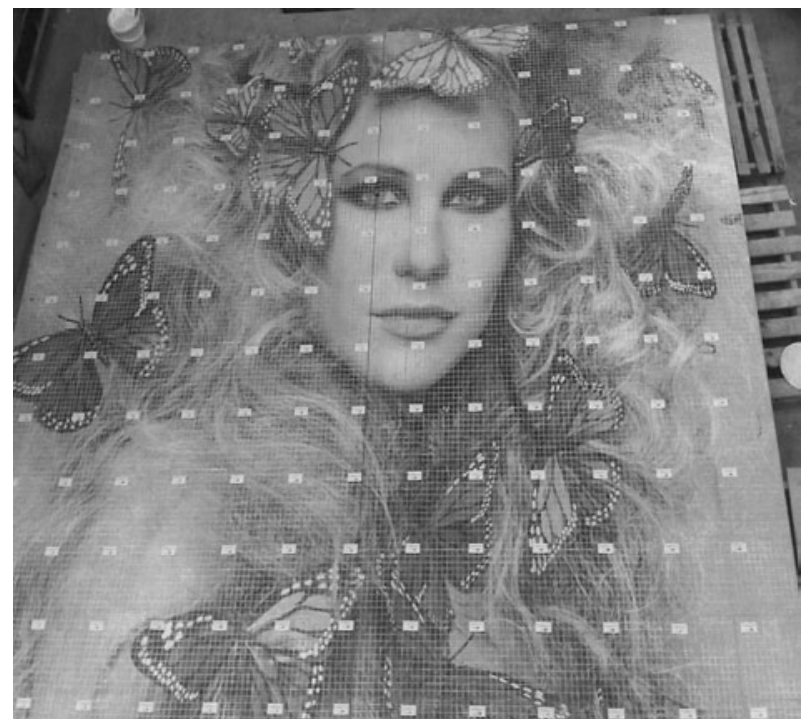

Mural confeccionado con teselas de vidrio sinterizado. 


\section{CONCLUSIÓNES}

- Este proyecto es innovador, porque partiendo de un producto ecológico, de cero emisiones, se aúnan tecnologías de última generación, como son los mosaicos translucidos por prensado y la decoración digital por inyección a unas dimensiones reducidas.

- Este producto abre nuevos mercados al mosaico vítreo, decoración de grandes superficies irregulares, domótica, sistemas de aviso, control en ambientes extremos, zonas de transito, salas ocio-multiusos, decoración vivienda interna-externa (creación de ambientes), húmedos (piscinas, jacuzzy, saunas, etc.).

- El desarrollo de esta nueva tecnología, ha permitido la manipulación y elaboración de paneles de mayor tamaño, pudiendo realizar diseños de dimensiones considerables ( $4 \mathrm{~m} \times 4,5 \mathrm{~m}$ ), tal como se puede observar en la foto adjunta y que se podrá apreciar en la feria de CEVISAMA 2011, en nuestro stand. Esto unido a la versatilidad de tamaño del mosaico vítreo, nos permite recubrir cualquier superficie irregular y conseguir efectos decorativos difícilmente realizables con cualquier otro material.

\section{BIBLIOGRAFIA}

1. J. H Conway, N. J. H Sloane, "Sphere Packings, Lattices and Groups" (Third Edition). ISBN 0-387-98585-9, 1988

2. J. E.Navarro, F.Negre : Materias primas para la preparación de esmaltes "Tecnología cerámica". Universidad de Valencia.(1985)

3. V. Caballer, J. Negre et al.. "Mejoría de la blancura de un vidriado mediante la aplicación de un diseño factorial de experimentos". Qualicer 1994. 6-9 Marzo. Castellon. España

4. T. Aste \& D. Weaire "The Pursuit of Perfect Packing" (Institute Of Physics Publishing London 2000) ISBN 0-7503-0648-3

5. N. J. A. Sloane, The Sphere Packing Problem, Plantilla:Arxiv (A technical survey from 2002).

6. Sacmi Imola; Prensado Cap. III, “Tecnología Cerámica Aplicada”. ISBN:8487683-29-0.

7. Sacmi Imola; Cocción Cap. VI, “Tecnología Cerámica Aplicada”. ISBN:8487683-29-0.

8. Timoshenko S., Strength of Materials, 3rd edition, Krieger Publishing Company, 1976, ISBN 0-88275-420-3

9. J. L Amoros J.L, M M .J Orts, V.Sanz, A.Escardino; “Prensado unidireccional de polvos cerámicos aglomerados por atomización-II-III Mecanismo de Compactación" en Ciencia y tecnología de los materiales cerámicos y vítreos. p. 133-144. Faenza Editrice Iberica S.L. Castellón.(1990).ISBN:8487683-00-2. 